\title{
Frequency analysis of multiperiodic $\delta$ Scuti stars
}

\author{
L. Mantegazza ${ }^{1}$, E. Poretti ${ }^{2}$, F.M. Zerbi ${ }^{1}$ \\ 'Dipartimento di Fisica Nucleare e Teorica, Università di Pavia, Italy \\ ${ }^{2}$ Osservatorio Astronomico di Brera, Merate, Italy
}

We report some of the recent results of our studies on $\delta$ Scuti star pulsation which are based on observations made at Merate and La Silla Observatories, sometimes in two site campaigns. Our recent experiences on X Caeli and 44 Tauri have shown that quite complicate light curves can be resolved even with observations obtained from one site only, if compact and accurate datasets are collected on sufficiently long time baselines (Mantegazza and Poretti, 1992; Poretti et al., 1992). Here we report the preliminary results of the light curve analysis of three more stars surveyed for more than 120 hours each: BI CMi, HD 18878 and HD 224639.

BI CMi has been observed in a two-site campaign in January-February 1991, the observations being collected during 17 nights. The frequency analysis of the light curve allowed us to identify unambiguously 4 pulsation modes at $8.247,8.863,8.514$ and $7.424 \mathrm{c} / \mathrm{d}$ with semi-amplitudes of $22,19,5$ and $5 \mathrm{mmag}$ respectively. Even if these components explain almost $90 \%$ of the data variance, there is evidence of the presence of other components of smaller amplitude $(<2.5 \mathrm{mmag})$, whose correct identification is however more ambiguous. The period ratios between the 4 frequencies evidences that at least some of them are ascribable to non-radial pulsations.

HD 18878 was observed from November 1991 to January 1992 at Merate Observatory, our data revealing clearly a multiperiodic behaviour. A preliminar frequency analysis yields four predominant frequencies (amplitudes ranging from 23 to $5 \mathrm{mmag}$ ): $6.86,11.22,11.18$ and $10.01 \mathrm{c} / \mathrm{d}$. The fit with these four frequencies leaves a rms residual of $7.3 \mathrm{mmag}$ and explains the $87 \%$ of the data variance. Further analysis is necessary to unambiguously evidence the other periodic terms with very small amplitudes present in the light curves.

HD 224639 has been previously observed by us in 1989 (Mantegazza and Poretti, 1990) in a short two-site campaign. Those data were insufficient to allow a light curve solution, and therefore new observations were collected by us at La Silla Observatory in September and October 1991 during 20 nights. The data frequency analysis is now in progress. As a preliminary result we can report that the star has a very rich pulsational spectrum with 8 components with semi-amplitudes between 20 and 5 mmag, and some more with smaller ones.

\section{References:}

Mantegazza, L. and Poretti, E., 1990, in: Confrontation between stellar pulsation and evolution, Astr.Soc. of Pacific Conf.Series 11, p 324.

Mantegazza, L., Poretti, E., 1992, Astron.Astrophys. 255, 153.

Poretti, E., Mantegazza, L., Riboni, E., 1992, Astron.Astrophys. 256, 113. 